\title{
The semiclassical helium atom
}

\author{
D. Wintgen, K. Richter, and G. Tanner \\ Fakultät für Physik der Universität, Hermann-Herder-Str. 3, 7800 Freiburg, Germany
}

(Received 12 November 1991; accepted for publication 13 January 1992)

Recent progress in the semiclassical description of two-electron atoms is reported herein. It is shown that the classical dynamics for the helium atom is of mixed phase space structure, i.e., regular and chaotic motion coexists. Semiclassically, both types of motion require separate treatment. Stability islands are quantized via a torus-quantization-type procedure, whereas a periodic-orbit cycle expansion approach accounts for the states associated with hyperbolic electron pair motion. The results are compared with highly accurate $a b$ initio quantum calculations, most of which are reported here for the first time. The results are discussed with an emphasis on previous interpretations of doubly excited electron states

\section{INTRODUCTION}

The failure of the Copenhagen School to obtain a reasonable estimate of the ground-state energy of the helium atom (see e.g. the old review by van Vleck ${ }^{1}$ ) and of the $\mathrm{H}_{2}^{+}-$molecule ${ }^{2}$ was a cornerstone in the evolution of quantum mechanics. The pessimistic point of view concerning a semiclassical treatment of two-electron atoms is summarized in the book of Born ${ }^{3}$ :

"... the systematic application of the principles of the quantum theory ... gives results in agreement with experiment only in those cases where the motion of a single electron is considered; it fails even in the treatment of the motion of the two electrons in the helium atom.

This is not surprising, for the principles used are not really consistent... A complete systematic transformation of the classical mechanics into a discontinuous mechanics is the goal towards which the quantum theory strives."

Nowadays we know the essential shortcomings of the old quantum theory:

(i) the role of conjugate points along classical trajectories and their importance for the approach to wave mechanics (which was not developed at those times) were not properly accounted for; and

(ii) the precise role of periodic trajectories when the classical dynamics are non-integrable or even chaotic was unknown.

The pessimistic point of view dominated the research for several decades and there were no successful attempts to attack the problem until Leopold and Percival ${ }^{4}$ in 1980 gave a reasonable estimate of the ground-state energy of the helium atom, ignoring however item (ii). Nowadays, a proper semiclassical treatment of the helium atom is still an outstanding problem of the semiclassical theory. The helium atom therefore remains the essential touchstone of semiclassical mechanics, even though considerable progress in the development of the formal theory has been achieved within the last years, most of which is documented in this issue of CHAOS.

A semiclassical description of two-electron atoms is also highly desirable, because most parts of the spectral regions are still unexplored, both experimentally and quantum theoretically. From a conceptual point of view highly accurate quantum calculations are not too difficult to perform. However, the high dimensionality of the problem combined with the vast density of states can make the calculations cumbersome and elaborate. In addition, one has to deal with singular potentials, long-ranged interactions, and typically many open decay-channels, all of which prevents the success of brute-force methods. Furthermore, the problem of understanding the structure of the quantum solutions still remains after solving the Schrödinger equation. Again, simple interpretation of classical and semiclassical methods assists in illuminating the structure of the solutions. Classical calculations may also help to uncover local integrals of motions or adiabatic coordinates. Exploiting such properties may facilitate (approximate) quantum calculations considerably.

The necessary ingredient for any semiclassical analysis is a proper understanding of the underlying classical dynamics. Unfortunately, this information is highly nontrivial to obtain. The equations of motion are multidimensional, non-integrable, and singular, hence far away from an easy-to-do-job. In addition, the independent particle case $1 / Z=0$ ( $Z$ is the nuclear charge) is highly degenerate, which prohibits an application of the KAM theory to derive an independent particle limit. In other words, the phase space structure of the hydrogenic motion of two independent electrons depends on an (infinitesimal) perturbation and not only on the zero-order Hamiltonian itself. As a matter of fact, until recently it was even unknown whether the motion of two-electron atoms is ergodic or not. $^{5}$

In this contribution we review on recent progress in the classical and semiclassical description of two-electron atoms. We show that the classical phase space is of mixed structure, i.e. regular and irregular motion of the electron pair co-exist. Roughly, the angular type of motion (i.e. bending motion of the electron pair relative to the nucleus) is mostly stable, whereas radial motion is mostly (but not always) unstable. The radial instability typically leads to ionization of one electron (we restrict the analysis to energies below the three-particle breakup threshold). A 
semiclassical treatment has to distinguish between fully stable (i.e. stable in all dimensions) and (partly) unstable motion. Fully stable motion allows for approximate torus quantization, and this applies to electron pair motion, where both electrons are located on the same side of the nucleus in a near-collinear configuration. ${ }^{6}$ The classical motion for near-collinear configurations with both electrons on different sides of the nucleus turns out to be fully chaotic. In this case the semiclassical Gutzwiller theory combined with the cycle expansion method yields good results. ${ }^{7}$

We will not discuss in this contribution how to solve the Schrödinger equation, even though we partly "review" on quantum results which are at present not available in the literature. Our main concern lies in demonstrating the power of semiclassical methods for two-electron atoms and how the semiclassical results compare with highly accurate quantum results.

\section{CLASSICAL MOTION IN HELIUM}

There are only few rigorous results about the general classical three-body Coulomb problem. The reason for the lack of popularity of quantitative classical studies is obvious: the equations of motion are multi-dimensional; nonintegrable, and singular. In addition, the independent particle case $1 / Z=O(Z$ is the nuclear charge) is highly degenerate, which prohibits a direct application of the KAM theorem to derive a proper independent particle limit. Quantitative analyses of the problem are being developed at present. ${ }^{5-11}$

An essential ingredient for the classical analysis of the three-body Coulomb problem is the regularization of the equations of motion. ${ }^{12}$ For a nucleus with charge $Z$ and infinite mass the Hamiltonian reads (atomic units used, $e=m_{e}=1$ ):

$$
H=\frac{\mathbf{p}_{1}^{2}+\mathbf{p}_{2}^{2}}{2}-\frac{Z}{r_{1}}-\frac{Z}{r_{2}}+\frac{1}{r_{12}} .
$$

The electron-nucleus distances are given by $r_{i}, i=1,2$, and the distance between the electrons is $r_{12}$. Whenever an inter-particle distance vanishes (particle collision) the potential energy diverges. There is a striking difference in the topology of the various collisions. In analogy to the motion of the electron in the hydrogen atom, the motion can be regularized for binary collisions, where only one interparticle distance vanishes. However, the triple collision $r_{1}$ $=r_{2}=r_{12}=0$ cannot be regularized, i.e. these solutions have branch points of infinite order. ${ }^{13} \mathrm{~A}$ numerically convenient method to regularize the binary collisions can be found in Ref. 5.

The energy $E$ and the total angular momentum $\mathbf{L}$ are constants of motion. Furthermore, the Hamiltonian (1) is invariant under reflection $\left(\mathrm{r}_{1}, \mathbf{r}_{2}\right) \rightarrow\left(-\mathbf{r}_{1},-\mathbf{r}_{2}\right)$ and particle exchange $\left(\mathbf{r}_{1}, \mathbf{r}_{2}\right) \rightarrow\left(\mathbf{r}_{2}, \mathbf{r}_{1}\right)$. The Hamiltonian (1) is homogeneous in coordinates and momenta and the equations of motion can be scaled to energy independent form. The accumulated action along a classical path is then
$\tilde{S(E)}=2 \pi z S$ with $z=(-E)^{-1 / 2}$ and $2 \pi S$ the action at energy $E=-1$.

Here we shall focus on total angular momentum $\mathbf{L}$ $=0$, for which the motion of the electrons is confined to a plane fixed in configuration space. This removes three of the total of six degrees of freedom, and we take the three inter-particle distances $r_{i}$ as dynamical variables. It is convenient to replace these by the perimetric coordinates ${ }^{14}$

$x=r_{1}+r_{2}-r_{12}, y=r_{1}-r_{2}+r_{12}, z=-r_{1}+r_{2}+r_{12}$.

with $x, y, z \geqslant 0$. The perimetric coordinates treat all interparticle distances democratically. The discrete symmetries of the Hamiltonian (1) are readily identified as symmetry planes in the perimetric coordinate set. Collinear motion with both electrons on different sides of the nucleus is confined to the $x \equiv 0$ plane. Collinear motion with both electrons localized on the same side of the nucleus is given by either $y \equiv 0$ or $z \equiv 0$. Finally, motion on the so-called Wannier ridge ${ }^{15} r_{1} \equiv r_{2}$ takes place in the $y \equiv z$ plane. The electron motion in the symmetry planes becomes essentially two-dimensional. The third degree of freedom is taken into account by linearizing the equations of motion around the symmetry plane.

Here we will focus on near-collinear configurations only, but as we will see this is already enough to uncover the difficulties of the full problem and to draw some definitive conclusions about it. Our main concern is to unravel the structure and the organization of the periodic orbits. They are the main ingredients of modern multidimensional semiclassical theories as discussed in the next sections. The radial motion along the Wannier ridge of symmetrical electron configurations $r_{1} \equiv r_{2}$ is (except for the so-called Langmuir orbit ${ }^{5}$ ) extremely unstable and of minor importance for a semiclassical treatment. Therefore we will discuss this type of classical motion only briefly.

\section{A. The $Z^{2+} e^{-} e^{-}$configuration}

Consider a collinear arrangement of a nucleus of charge $Z$ and of two electrons, both being on the same side of the nucleus. The fundamental periodic motion of such a configuration is a coherent oscillation of both electrons with the same frequency but, as it turns out, with large differences in their individual radial amplitudes and velocities as shown in Fig. 1(a) for $Z=2$ helium. The outer electron appears to stay nearly frozen at some fixed radial distance. For this reason we label the orbits as frozen planet. configurations. The minimal nuclear charge to bind an electron in this type of collinear configuration is $Z>1$; otherwise the outer electron potential is purely repulsive. On the other hand, $1 / Z$ must be non-zero, i.e. the repulsive electron-electron interaction is of crucial importance for the formation of these states. Thus the configurations considered here cannot be described within an independent particle model and are of non-perturbative nature. The high degree of classical dynamical localization of the outer electron is mostly pronounced for helium and becomes weaker for larger integer values of $Z$. 


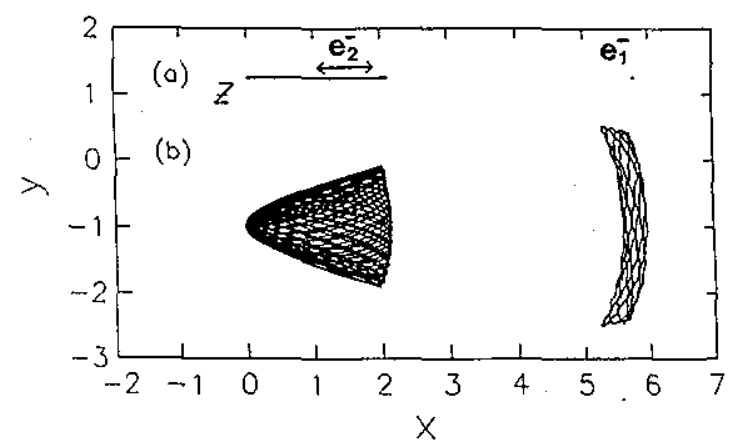

FIG. 1. (a) The straight line motion of the electron pair for the frozen planet $\mathrm{PO}$, while a nonperiodic but regular trajectory in its neighborhood is shown in (b). Energy scaled units $(-E) r$ are used.

Remarkably, the periodic orbit (PO) of Fig. 1(a) is linearly stable with respect to variations in the initial conditions. This is demonstrated in Fig. 1(b) which shows the resulting (regular) motion of the electrons when they are initially in a slightly off-collinear arrangement. The inner electron moves on perturbed Kepler ellipses around the nucleus, while the outer electron remains trapped at large radial distances following the slow angular oscillations of the inner electron.

For collinear configurations the motion is confined to the three-dimensional energy shell of a four-dimensional subspace of the full phase space. It is convenient to visualize the phase space structure by taking Poincaré surfaces of section. Such a section is shown for helium in Fig. 2. The phase space position $\left\{r_{1}, p_{1}\right\}$ of the outer electron is monitored each time the inner electron approaches the nucleus $\left(r_{2}=0\right)$. The PO shown in Fig. 1(a) appears as the elliptic fixed point in the center of the extended torus structure. Near the fixed point the motion of the outer electron is nearly harmonic, but for large radial distances the tori are deformed according to the almost Keplerian motion of the outer electron. The non-closed manifolds surrounding the

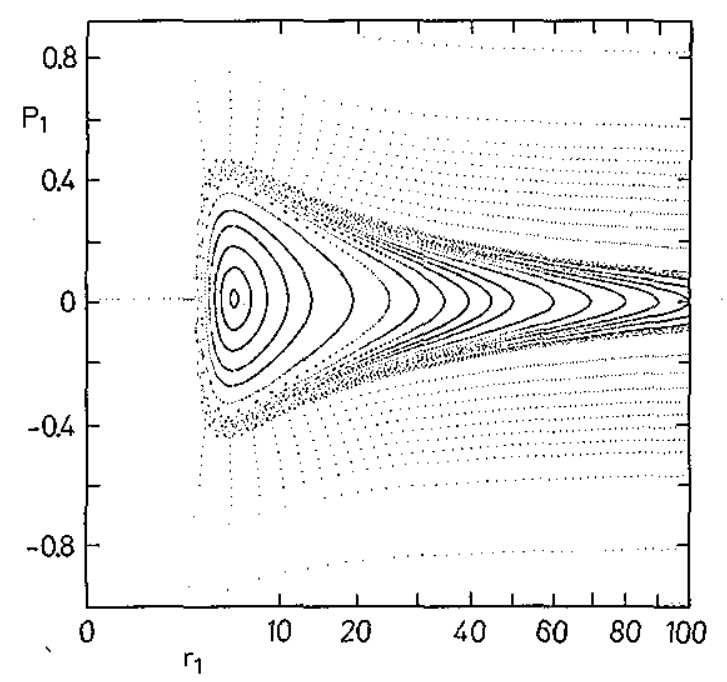

FIG. 2. Poincaré surface of section $\left(r_{2}=0\right)$ for collinear configurations with both electrons on the same side of the nucleus.

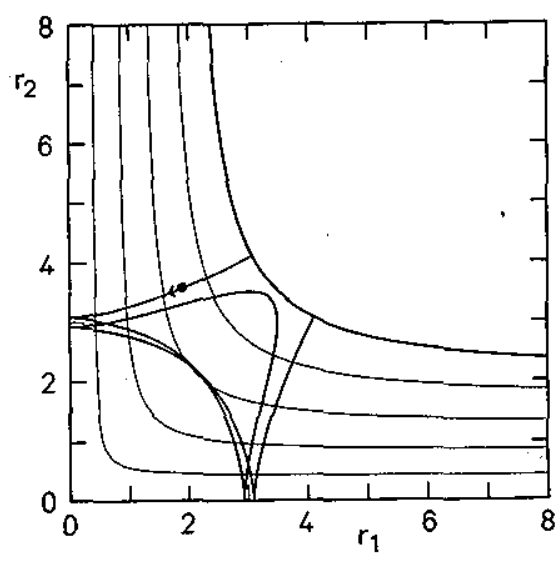

FIG. 3, Equipotential lines and boundary of the classical allowed region for the collinear electron configuration with both electrons on opposite sides of the nucleus $(Z=2, E=-1)$. The PO ' +--- ' is also shown.

tori represent (regular) trajectories for which the outer electron ionizes with $p_{1} \rightarrow\left(2 E_{1}+2 / r_{1}\right)^{1 / 2}\left(E_{1}\right.$ is the asymptotic excess energy of the ionizing electron). Recalling the additional stability of the bending degree of freedom (i.e. motion off the collinear arrangement), the fundamental PO of Fig. 1 is embedded in a fully six-dimensional island of stability in phase space.

The near-integrability of the three-body Coulomb problem for asymmetric configurations as shown in Figs. 1 and 2 is a remarkable fact, which nevertheless was unknown until recently. The stability of the outer electron with respect to radial motion can be understood in a static model (e.g. by fixing the inner electron at its classical expectation value or its outer classical turning point) but the stability with respect to the bending degree of freedom is somewhat surprising and its origin is purely dynamical. It is also unexpected and surprising that these classical configurations are extremely stable against autoionization, which is allowed energetically. Intuitively, one would expect that the inner electron "kicks" the loosely bound outer electron out because the electron-electron interaction $1 / r_{12}$ is maximized in such a collinear configuration. However, as we will see in the next section, instabilities of the system emerge mostly from the (non-regularizable) triple collisions, where all inter-particle distances vanish.

\section{B. The $e^{-Z^{2}+} e^{-}$configuration}

Configurations where the electrons move on opposite sides of the nucleus are energetically favored because the electron-electron interaction is minimized. Quantum mechanically, these are the (resonant) states in which $-\langle\cos \Theta\rangle$ is close to unity. Here, $\Theta$ is the angle between $\mathbf{r}_{1}$ and $\mathbf{r}_{2}$. These states are dominantly excited in singlephoton transitions from the ground state. ${ }^{16}$

Equipotential lines for this type of collinear electron arrangement are shown in Fig. 3 together with a typical periodic trajectory. The system ionizes if either $r_{1} \rightarrow \infty$ or $r_{2} \rightarrow \infty$. As a matter of fact, the topology of the equipotential lines and of the boundary of the classically allowed 
region do not depend on the details of the underlying particle-particle interactions. The main characteristic of the potential surfaces is that there are two alternative ways of the system to ionize, i.e. only one particle (or equivalently one degree of freedom) can ionize whereas the other particle remains bounded. Similar potential surfaces can be found in problems such as the hydrogen atom in a uniform magnetic field ${ }^{17}$ (where the electron can ionize either parallel or anti-parallel to the magnetic field), the motion of triatomic molecules for energies below the three-particle breakup threshold, ${ }^{18}$ the $x^{2} y^{2}$ potential, ${ }^{19}$ or the motion of ballistic electrons in heterojunctions ${ }^{20}$ modeled by elastic pinball scattering of particles (such as the four-disk scattering system ${ }^{17}$ or the hyperbola billiard ${ }^{21}$ ).

The classical motion of the collinear helium atom with the electrons on different sides of the nucleus turns out to be fully chaotic, even though we cannot rigorously prove this. A system is called "chaotic" if all PO are linearly unstable and their number proliferates exponentially with the action (or some other length characteristic). The exponential proliferation becomes obvious if the PO can be mapped onto a tree of symbols as, e.g., for the anisotropic Kepler problem (AKP) ${ }^{2,22}$ or the diamagnetic Kepler problem (DKP). ${ }^{17}$ Our numerical findings on the collinear motion of the helium atom suggest that the PO obey a binary coding.

To characterize the motion on the potential surface, Fig. 3, we introduce a symbolic description of the trajectories by recording the sequence $\left\{i_{j}\right\}, \ldots, i_{-1}, i_{0}, i_{1}, i_{2}, \ldots$, of electron collisions with the nucleus, i.e., $r_{i}=0$. Starting at an end point in Fig. 3 the PO is then coded by the periodically continued string of symbols . . 12122121. . . There appears to be no restriction on allowed symbol sequences, but for a PO the length of the periodic symbol sequence must be even.

The restrictions on the possible POs can be overcome by de-symmetrizing the motion and considering the motion in the fundamental domain, ${ }^{17,23}$ which is only half of the configuration space shown in Fig. 3 with an elastically reflecting wall at $r_{1} \equiv r_{2}$. This classical procedure corresponds to the separation of discrete symmetries in quantum mechanics and to the symmetrization of the Green function in the semiclassical theory. Here, the discrete symmetry corresponds to the exchange of electron coordinates $r_{1} \leftrightarrow r_{2}$ (Pauli principle). All information (either classically or quantum mechanically) is contained in the de-symmetrized motion of the fundamental domain, ${ }^{24}$ to which we will restrict ourselves from now on.

When there is a discrete symmetry only an initial segment of the periodic orbit needs to be considered. After some fraction of its period, the orbit will pass through an image (under the discrete symmetry group) of the initial point. The further evolution may then be obtained from symmetry images of the initial segment. The action of the orbit and the period, being scalars, are simply additive under the symmetry transformation. The stability matrix, however, is sensitive to the type of symmetry transformation. ${ }^{24}$ An example for such a symmetric $P O$ is the trajectory shown in Fig. 3. A redefinition of the coding scheme accounts for the symmetry of the PO: a collision is denoted by the symbol " + ' if the previous collision was by the same electron, and by the symbol ' $\ldots$ ' if the collision before was by the other electron. Using this fundamental coding the symbol string for the PO reads ' $+\quad-\quad-\quad$ '. Now we have to add an image of the fundamental code to obtain the symbol string of the PO in the full domain, just as with the PO itself. The PO has (topological) length 4 , because its code consists of repetitions of a string of four symbols.

We now assume that the collinear PO not involving triple collisions can be mapped one-to-one onto the binary symbols $\{+,-\}$. This conjecture is supported by numerical results summarized in Table $I$, where we list all PO up to symbol length 6 . Some of these orbits are shown in Fig. 4.

Apart from the missing orbit " + ' (which parallels the AKP and the DKP problem) all PO exist for the symbol sequences of Table $\mathrm{I}$. The coding takes care automatically of the discrete symmetries of PO. The maximal number of conjugate points (within the collinear configuration) of a $\mathrm{PO}$ is given by its symbol length, and the Morse index $\alpha$ by twice the symbol length. The type of fixed point is determined whether the number of " - ' in the sequence is odd or even. All stability exponents $u$ listed in Table I are strictly positive, i.e. all the orbits are unstable with respect to the motion within the collinear arrangement (radial correlation). The linearized motion off the symmetry plane (angular correlation) is however stable and characterized by the winding number $\gamma$, i.e. the eigenvalues $\exp ( \pm 2 \pi i \gamma)$ of the stability matrix. ${ }^{24}$

The missing fundamental orbit (i.e., orbit of length 1) - + ' can be formally assigned to a PO for which one electron is removed to infinity whereas the second electron is moving on a degenerate Kepler ellipse. The orbit does not give rise to resonant structures which are formed within a finite reaction zone around the nucleus. A 'naive' WKB-quantization of this trajectory, however, gives the correct energies of the two-particle breakup thresholds, i.e. the (hydrogen-like) energies of the remaining bound electron.

An alternative way to introduce the coding is to exploit the discrete properties of the periodic orbits, particularly the Morse index. ${ }^{17,24}$ Each symbol of the alphabet $\{+$, - \} carries some discrete additive or multiplicative weight for the discrete properties of the PO. For example, each symbol is associated with one pair of self-conjugate points along the trajectory, which allows a definition of the coding via collisions with potential boundaries. ${ }^{17}$ Also, each ' - , symbol changes the sign of the trace of the orbit's stability matrix and the type of hyperbolicity, respectively. A symbolic description must be able to describe these discrete properties, otherwise it is useless.

The coding scheme introduced above of the collinear helium atom parallels that of the AKP. There, consecutive crossings of the electron with the symmetry axis are recorded, ${ }^{2,22}$ which also leads to a binary coding. The orbits coming from a collision with the nucleus (collision manifold) generate the partitioning of the phase space into 
TABLE I. Various properties of the collinear periodic orbits of the helium atom. $u$ is the stability exponent, i.e. the Liapunov exponent times the action $S$ of the orbit. The Morse index $\alpha$ for the motion in the symmetry plane and the winding number $\gamma$ for the linearized motion off the symmetry plane are given in the next columns. The type of fixed point (FX) is denoted by $\mathrm{H}$ for hyperbolic orbits and by IH for hyperbolic orbits with reflection (taken from Ref. 7).

\begin{tabular}{|c|c|c|c|c|c|c|c|c|c|c|c|}
\hline No & Code & & & & & & $S$ & $u$ & $\gamma$ & $\alpha$ & FX \\
\hline 1 & + & & & & & & $\ldots$ & $\ldots$ & $\ldots$ & 2 & $\mathrm{H}$ \\
\hline 2 & - & & & & & & 1.82900 & 0.6012 & 0.5393 & 2 & $I H$ \\
\hline 3. & + & - & & & & & 3.61825 & 1.8622 & 1.0918 & 4 & IH \\
\hline 4 & + & + & - & & & & 5.32615 & 3.4287 & 1.6402 & 6 & IH \\
\hline 5 & + & - & - & & & & 5.39452 & 1.8603 & 1.6117 & 6 & $\mathbf{H}$ \\
\hline 6 & + & + & + & - & & & 6.96677 & 4.4378 & 2.1710 & 8 & IH \\
\hline 7 & + & + & - & - & & & 7.04134 & 2.3417 & 2.1327 & 8 & $\mathbf{H}$ \\
\hline 8 & + & - & - & - & & & 7.25849 & 3.1124 & 2.1705 & 8 & IH \\
\hline 9 & + & + & + & + & - & & 8.56619 & 5.1100 & 2.6919 & 10 & IH \\
\hline 10 & + & + & + & - & - & & 8.64307 & 2.7207 & 2.6478 & 10 & $\mathrm{H}$ \\
\hline 11 & + & + & - & + & - & & 8.93700 & 5.1563 & 2.7292 & 10 & $\mathbf{H}$ \\
\hline 12 & + & + & - & - & - & & 8.94619 & 4.5932 & 2.7173 & 10 & IH \\
\hline 13 & + & - & + & - & - & & 9.02690 & 4.1765 & 2.7140 & 10 & IH \\
\hline 14 & + & - & - & - & - & & 9.07179 & 3.3424 & 2.6989 & 10 & $\mathrm{H}$ \\
\hline 15 & + & + & + & + & + & - & 10.13874 & 5.6047 & 3.2073 & 12 & IH \\
\hline 16 & + & + & + & + & - & - & 10.21674 & 3.0324 & 3.1594 & 12 & $\mathrm{H}$ \\
\hline 17 & + & + & + & - & + & - & 10.57067 & 6.1393 & 3.2591 & 12 & $\mathrm{H}$ \\
\hline 18 & + & + & + & - & - & - & 10.57629 & 5.6766 & 3.2495 & 12 & IH \\
\hline 19 & + & + & - & + & - & - & 10.70699 & 5.3252 & 3.2520 & 12 & IH \\
\hline 20 & + & + & - & - & + & - & 10.70699 & 5.3252 & 3.2520 & 12 & IH \\
\hline 21 & + & + & - & - & - & - & 10.74304 & 4.3317 & 3.2332 & 12 & $\mathrm{H}$ \\
\hline 22 & + & - & + & - & - & - & 10.87855 & 5.0002 & 3.2626 & 12 & $\mathbf{H}$ \\
\hline 23 & + & - & - & $\ldots$ & - & - & 10.91015 & 4.2408 & 3.2467 & 12 & IH \\
\hline
\end{tabular}

cells which are uniquely labeled by the binary code. Analogously, the collision manifold for the collinear helium atom is represented by the trajectories coming out of a triple collision, where all inter-particle distances vanish.

The collision manifold plays a peculiar role in the classical description of the collinear helium atom. If the singularity of the Coulomb potential is smoothed (for example by a non-vanishing total angular momentum $L$ of the three-body complex) the orbits starting and ending in a triple collision become periodic trajectories. The appropriate coding is then ternary and equivalent to the DKP problem ${ }^{17}$ and to problems having similar potential surfaces, as discussed at the beginning of this section. Eventually, if the smoothing is too strong, the symbolic tree of orbits is pruned and some orbits do no longer exist or become stable. The further fundamental orbit ' 0 ' of the ternary coding is the symmetric stretch motion of the electrons with $r_{1} \equiv r_{2}$. The (in-phase) symmetric stretch trajectory is better known in atomic physics as Wannier ridge configuration, where it plays an important role in the Wannier theory of three-particle breakup (for $E>0$ ).

In the Wannier configuration of non-vanishing angular momentum the electrons move on closed Kepler ellipses with $\mathbf{r}_{1}=-\mathbf{r}_{2}$. An important property of the Wannier orbit (as well as the other orbits of the collision manifold) is that its stability exponent diverges as it approaches the triple collision. ${ }^{5}$ This is shown in Fig. 5 where we plot (for various values of the nuclear charge $Z$ ) the stability index $\lambda_{\alpha}$ for the radial correlation against a logarithmic scale of the scaled total angular momentum. The diverging stability index for the Wannier orbit has important semiclassical consequences: the orbit does not give rise
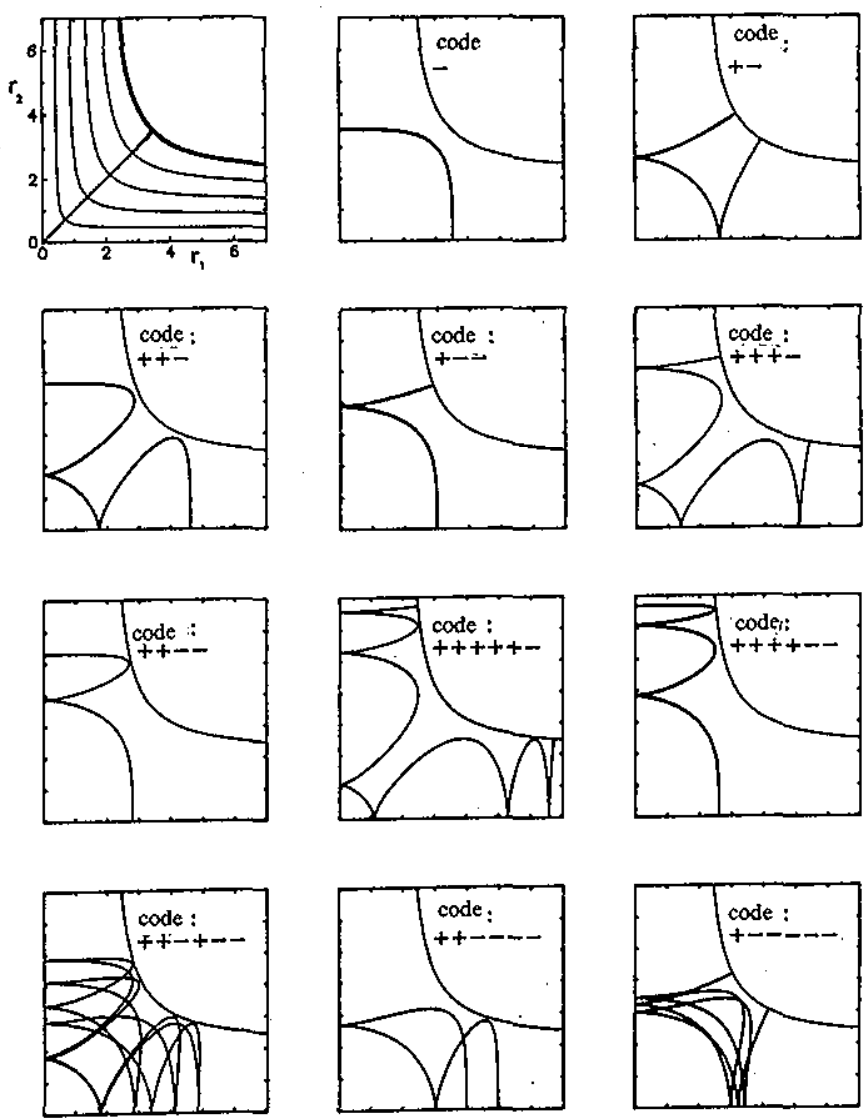

FIG. 4. Periodic orbits of the collinear helium atom $(\Theta=\pi)$. The upper left figure shows some equipotential lines and the symmetric stretch motion (Wannier configuration) along the symmetry line $r_{1} \equiv r_{2}$. 


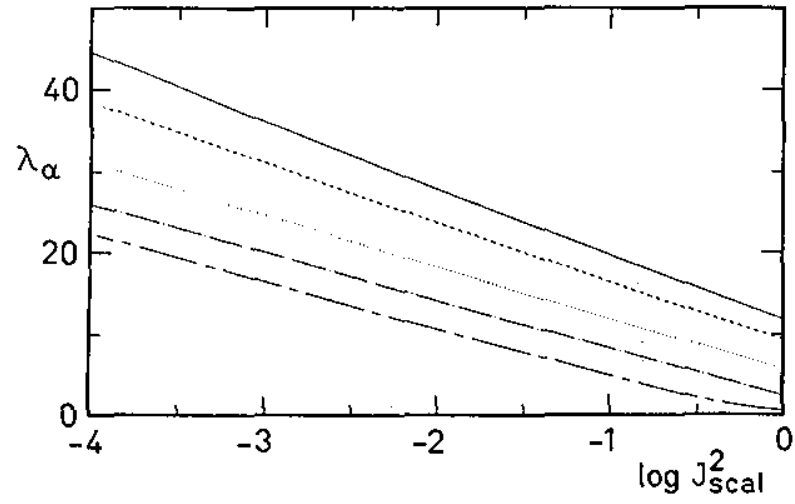

FIG. 5. The stability exponent $\lambda_{\alpha}$ for the Wannier PO as a function of the scaled total angular momentum $J_{\text {scal }}$ for various $Z$-values, $Z$ $=0.4,0.5,1,5,100$ (from above). The scaled angular momentum is related to the real angular momentum $L$ by $J_{\text {scal }}=L / L_{\max }$, where $L_{\max }$ $=2\left(Z-\frac{1}{4}\right) / \sqrt{-E}$ is the maximal angular momentum at energy $E$.

to resonant structures in the density of states (see also the section about semiclassical quantization). This classical prediction is more remarkable considering that since decades the credo of electron pair motion along the Wannier ridge plays an important role in the interpretation of doubly excited states. ${ }^{25-30}$ The absence of resonant structures related to the Wannier orbit does not contradict the Wannier theory of double electron escape in which $E \geqslant 0$ is assumed. Both electrons then escape to infinity in a symmetric configuration and they never return to the nucleus, which is the source of the enormous instability of the periodic Wannier orbit.

\section{SEMICLASSICAL QUANTIZATION}

\section{A. Periodic orbit theory}

The connection between the quantum eigenvalues and periodic orbits was obtained by Gutzwiller ${ }^{2,31}$ starting from the relation between the density of states $\rho$ and the trace of Green function $G, \rho(E)=-(1 / \pi) \operatorname{Im} \operatorname{tr} G$. The Green function is the Fourier transform of the propagator $K$,

$$
G\left(\mathbf{q}_{2}, \mathbf{q}_{1} ; E\right)=\frac{1}{i \hbar} \int d t K\left(\mathbf{q}_{2}, \mathbf{q}_{1} ; t\right) e^{i E t / \hbar}
$$

Consistent with the semiclassical approximation all integrals are evaluated using the stationary phase approximation. ${ }^{32}$ For the propagator one then has an approximation in terms of classical paths connecting $\mathbf{q}_{1}$ and $\mathbf{q}_{2}$ in time $t$,

$$
\begin{aligned}
K\left(\mathbf{q}_{2}, \mathbf{q}_{1} ; t\right)= & (2 \pi i \hbar)^{-N / 2} \sum_{\text {paths }}\left|D_{W}\right|^{1 / 2} \times \exp \left(i W_{p, 21} / \hbar\right. \\
& \left.-i \pi v_{p} / 2\right)
\end{aligned}
$$

with $W_{p, 21}=\int_{1}^{2} L d t$ the Lagrangian action,

$$
D_{W}=\operatorname{det}\left(-\frac{\partial^{2} W_{p, 21}}{\partial \mathbf{q}_{2} \partial \mathbf{q}_{1}}\right)
$$

the determinant of second derivatives, and $v_{p}$ the number of caustics, i.e. it counts the number of zeros of the determinant (5) along the path $p$.

A semiclassical expression for the Green function is the integral (3) with the (exact) propagator replaced by its semiclassical approximation (4). Stationary phase approximation of the Fourier integral yields

$$
\begin{aligned}
G\left(\mathbf{q}_{2}, \mathbf{q}_{1} ; E\right)= & G_{0}+\frac{1}{i \hbar(2 \pi i \hbar)^{(N-1) / 2}} \sum_{p}\left|D_{S}\right|^{1 / 2} \\
& \times \exp \left[i S_{p}(E) / \hbar-i \pi v_{p}^{\prime} / 2\right],
\end{aligned}
$$

where now the sum extends over classical paths of nonvanishing length connecting $\mathbf{q}_{1}$ and $\mathbf{q}_{2}$ at a fixed energy $E$, irrespective of the time it takes; $S_{p}$ is the classical action $\int_{p} \mathbf{p} d \mathbf{q}$ of Maupertuis,

$$
D_{S}=\operatorname{det}\left(\begin{array}{ll}
\frac{\partial^{2} S_{p}}{\partial \mathbf{q}^{\prime} \partial \mathbf{q}} & \frac{\partial^{2} S_{p}}{\partial E \partial \mathbf{q}} \\
\frac{\partial^{2} S_{p}}{\partial \mathbf{q}^{\prime} \partial E} & \frac{\partial^{2} S_{p}}{\partial E^{2}}
\end{array}\right)
$$

is the determinant of second derivatives, and the index $v_{p}^{\prime}$ counts the number of caustics on the energy shell (which, dependent on the sign of $\partial^{2} W / \partial t^{2}$, may differ from $v_{p}$ by unity). The paths of zero length contribute differently and are contained in $G_{0}$. They are unimportant for the following discussion.

Finally, to obtain the density of states we have to integrate the diagonal elements of the Green function over position space. The phase of $G$ is stationary if the final and initial momenta coincide, which is the condition that the trajectory be periodic. In the neighborhood of every closed path a coordinate system with $q_{1}$ along the path and $q_{2}, \cdots q_{N}$ perpendicular to it may be introduced. Using the factorization of the determinant $D_{S}$ and the fact that up to second order in the deviations from the trajectory the action only depends on the stability matrix of the classical path, one finds

$$
\begin{gathered}
\frac{1}{(2 \pi i \hbar)^{(N-1) / 2}} \int d q_{2} \cdots d q_{N}\left|D_{S_{p}}\right|^{1 / 2} \\
\times \exp \left[i S_{p}(\mathbf{q}) / \hbar-i v_{p}^{\prime} \pi / 2\right] \\
=\frac{1 \exp \left(i S_{p} / \hbar-i \mu_{p} \pi / 2\right)}{\left|\dot{q}_{1}\right||\operatorname{det}(M-1)|^{1 / 2}}
\end{gathered}
$$

where $S_{p}$ is the action along the periodic orbit, $M$ is the stability matrix around the orbit, and the phase shift $\mu_{p}$ is the Morse index of the PO. ${ }^{24}$

Since the trace of the stability matrix is independent of the position along the path, there remains the integral $\int d q_{1} / \dot{q}_{1}$, which by $d q / \dot{q}=d t$ is the period of the orbit. Allowing for multiple traversals of a particular PO, we finally find for the contribution of one primitive (i.e. nonrepeated) periodic orbit (PPO)

$$
\frac{-i}{\hbar} T_{p} \sum_{r=1}^{\infty} \frac{e^{\left(i S_{p} / \hbar-i \mu_{p} \pi / 2\right) r}}{\left|\operatorname{det}\left(M^{r}-1\right)\right|^{1 / 2}}
$$


The trace over $G_{0}$ can be calculated as an asymptotic series in powers of $\hbar^{33}$ The leading term is given by the size of the energy shell,

$$
\operatorname{tr} G_{0}=\int \frac{d \mathbf{p} d \mathbf{q}}{h^{N}} \delta[E-H(\mathbf{p}, \mathbf{q})]
$$

This function depends smoothly on energy.

\section{B. Quantization of elliptic islands: the frozen planet configurations}

As shown in the previous section, the frozen planet periodic orbit is linearly stable for helium. The two pairs of eigenvalues of the stability matrix $M$ are then complex numbers on the unit circle, i.e. $\lambda_{R}=\exp \left( \pm 2 \pi i \gamma_{R}\right)$ and $\lambda_{\odot}=\exp \left( \pm 2 \pi i \gamma_{\Theta}\right)$. For trajectories close to the periodic orbit the frequency ratios of the radial and angular motion transverse to the periodic orbit are given by the winding numbers $\gamma_{R}=0.0677$ and $\gamma_{\Theta}=0.4616$, respectively. Expanding the determinant into geometric series, the contribution (9) of the frozen planet periodic orbit to the density of states is

$$
\begin{aligned}
\rho_{f f o} \sim & \sum_{r=1}^{\infty} \sum_{k, l=0}^{\infty} \exp 2 \pi i r\left[\frac{S}{\hbar}-\frac{\mu^{\prime}}{4}-\left(l+\frac{1}{2}\right) \gamma_{R}\right. \\
& \left.-2\left(k+\frac{1}{2}\right) \gamma_{\Theta}\right],
\end{aligned}
$$

where the number of conjugate points along the trajectory are already contained in the winding number. The additional phase shift $\mu^{\prime}=2$ comes from the singularities in the Green function which are related to the vanishing total velocity at the turning points of the electrons and to binary collisions, for which the Jacobian of the transformation from (six-dimensional) Euclidean coordinates to an appropriate internal coordinate set [e.g. the perimetric coordinates (2)] vanishes. The non-Euclidean character of the internal coordinates is also responsible for the additional factor of 2 appearing for the contribution of the motion $\Theta$ perpendicular to the symmetry plane. ${ }^{31}$

The sum over the repetitions $r$ in Eq. (11) is a geometric series which can be summed analytically. Thus Eq. (11) yields a triple- WKB formula with three quantum numbers $n, k, l$,

$$
S(E)=2 \pi \hbar\left[n+\frac{1}{2}+\left(l+\frac{1}{2}\right) \gamma_{R}+(2 k+1) \gamma_{\Theta}\right] .
$$

Using the classical scaling property for the action and rearranging Eq. (12) results in a triple-Rydberg formula for the energies converging to the three-body breakup threshold,

$$
E_{n k l}=-\frac{S^{2}}{\left[n+\frac{1}{2}+\left(l+\frac{1}{2}\right) \gamma_{R}+(2 k+1) \gamma_{\Theta}\right]^{2}},
$$

with $S=1.4915$ the scaled action of the periodic orbit. The semiclassical quantum numbers $n, k$ and $l$ reflect the approximate separability of the associated semiclassical wave functions in the local coordinates $\left\{q_{i}\right\}$ of the periodic orbit. Nodal excitations along the orbit are described by $n$, whereas $k$ and $l$ count the excitations perpendicular to the orbit.

Before applying Eq. (12) or (13) blindly, one should realize the inherent approximations and restrictions of these formulas. For integrable systems the equations actually represent an approximation of the torus- (or EBK-) quantization procedure, where the actions of the irreducible circuits on the tori are quantized separately. ${ }^{34}$ Here these actions are approximated harmonically through the properties of the fixed point (periodic orbit) in the center of the elliptic island. The advantage of such an approach is that it is also applicable for non-integrable systems as long as the elliptic island surrounding the periodic orbit is large enough to support many eigenstates, i.e. its phase space volume is large compared to $(2 \pi \hbar)^{N}$. The obvious disadvantage is that we cannot expect the approximations to be of good quality if the phase space volume of the island is small, or if the phase space structure (e.g. non-elliptic deformations) varies strongly over small phase space distances. However, due to the scaling properties for the present system such restrictions limit the applicability only to transversal excitations $k, l$, but not to $n$. Roughly, the maximal meaningful values for $k$ and $l$ increase proportional to $\sqrt{n}$ because the nodal structures of the wave functions parallel and perpendicular to the orbit scale with $1 / \hbar$ and $1 / \sqrt{\hbar}$, respectively ${ }^{35}$ (due to the scaling property $n$ takes over the role of $1 / \hbar)$.

In the derivation of the triple-WKB formula (12) we consistently expanded all expressions to leading order in $\hbar$. We then expect the absolute semiclassical error to be of the order $\hbar^{2}$ and the relative error to be of the order $\hbar / n$ (for $n \gg k, l)$,

$$
S\left(E_{n k l}\right) / 2 \pi \hbar[n+c(k, l)]=1+\beta \frac{\hbar}{n},
$$

which shows the semiclassical limit $\hbar \rightarrow 0$ to be equivalent to $n \rightarrow \infty$. No general theory is available at present to estimate the error constant $\beta$. Nevertheless, the energy eigenvalues predicted with the simple semiclassical formula (13) should become exact in the semiclassical limit of high excitations with an error vanishing proportional to $n^{-4}$.

Note that the triple-Rydberg formula (13) yields real energies. In the lowest semiclassical approximation presented here the wave functions are square integrable and represent exactly bound states. These states can autoionize semiclassically by dynamical tunneling, ${ }^{36}$ but the decay widths for such processes decrease exponentially with the nodal excitation along the orbit. The formula applies to both symmetrical and antisymmetrical states of electron exchange (i.e. to the spectroscopic ${ }^{2 S+1} L^{\pi}={ }^{1} S^{e}$ and ${ }^{3} S^{e}$ series). Again, dynamical tunneling lifts this doublet degeneracy and the exchange energies vanish exponentially, but the precise determination of the splitting is beyond the scope of the lowest order semiclassical treatment.

Table II summarizes the positions and widths of frozen planet resonances $(n, k=0, l=0)$ with $n$ ranging from 2 to 10 together with the predictions of the simple semiclassical formula (13). Considering the rather large basis sets nec- 
TABLE II. Energies $E_{n k l}$ and total decay widths $\Gamma / 2$ for planetary states with total angular momentum $L=0$ and nodal quantum numbers $k$ $=l=0$. They are given for both symmetry classes ${ }^{i} S$ and ${ }^{3} S$. The predictions of the semiclassical formula (13) are given as $E_{s c t}$.

\begin{tabular}{|c|c|c|c|c|c|}
\hline \multirow[b]{2}{*}{$n$} & \multicolumn{2}{|c|}{$S^{c}$} & \multicolumn{2}{|c|}{${ }^{3} S^{e}$} & \multirow[b]{2}{*}{$-E_{s c l}$} \\
\hline & $-E$ & $\Gamma / 2$ & $-E$ & $\Gamma / 2$ & \\
\hline 2 & 0.25737161 & 0.00001057 & 0.24996461 & 0.00000678 & 0.24792 \\
\hline 3 & 0.14106415 & 0.00001163 & 0.14008848 & 0.00000440 & 0.13935 \\
\hline 4 & 0.08957080 & 0.00000202 & 0.08946782 & 0.00000017 & 0.089145 \\
\hline 5 & 0.06205355 & 0.00000056 & 0.06204127 & 0.00000003 & 0.061887 \\
\hline 6 & 0.04553866 & 0.00000020 & 0.04553924 & 0.00000037 & 0.045458 \\
\hline 7 & 0.03484264 & 0.00000036 & 0.03484385 & 0.00000014 & 0.034798 \\
\hline 8 & 0.02751759 & 0.00000118 & 0.02751928 & 0.00000002 & 0.027491 \\
\hline 9 & 0.02228457 & 0.00000054 & 0.02228366 & 0.00000003 & 0.022265 \\
\hline 10 & 0.01841198 & 0.00000005 & 0.01841189 & 0.00000003 & 0.018400 \\
\hline
\end{tabular}

essary to obtain the accurate quantum results (up to $\sim 5500$ basis states used) the accuracy of the semiclassical results, which are obtained on a pocket calculator, are rather impressive. In Fig. 6 we plot the semiclassical error for the quantum defect-like quantity $\mu_{m}$,

$$
\mu_{n}=N_{\text {eff }}-n,
$$

where the effective quantum number $N_{\text {eff }}$ is defined as the denominator in Eq. (13), i.e.

$$
N_{\text {eff }}=S / \sqrt{-E} \text {. }
$$

From Fig. 6 we deduce the leading term of the semiclassical error in the quantum defect $\mu_{n}$ to be $\beta=$ -0.0366 . Hence, the error is of the order of what we expected, but the prefactor $\beta$ is rather small.

As predicted by the semiclassical theory, the energy splittings $\Delta E_{ \pm}$between the parity doublets listed in Table II indeed decrease exponentially. The number of equal significant digits roughly increase by one for each additional node $n$. The widths of the resonances also decrease exponentially but they fluctuate rather largely around this general trend. The exponential stability of the quantum states is remarkable considering the vastly increasing number of open channels into which the states can decay; the $(n, k, l)=(10,0,0)$ state, e.g., is coupled to 55 continuum

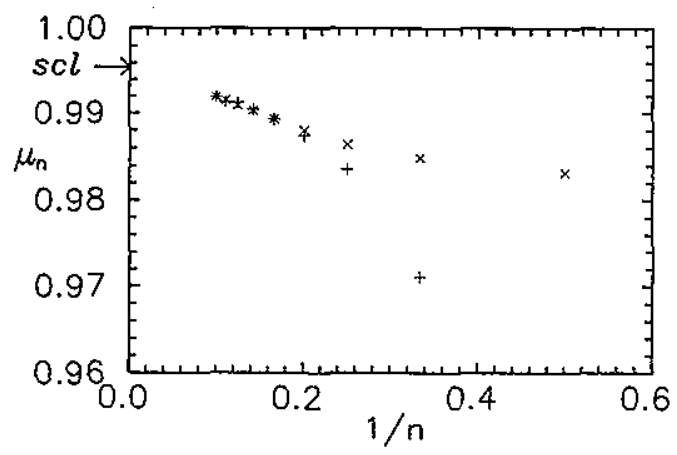

FIG. 6. Quantum defect $\mu_{n}$ as defined by Eq. (15). Both symmetry classes, ' $S^{e}(+)$ and ${ }^{3} S^{e}(X)$, are shown. The semiclassical limit is marked by an arrow. channels. The extreme stability against (non-radiative) decay is a direct consequence of the semiclassical nature of these states.

A direct examination of the nodal structure of the associated wave functions is a more stringent test than comparing energy eigenvalues. Figure 7 (a) depicts the condi- (a)

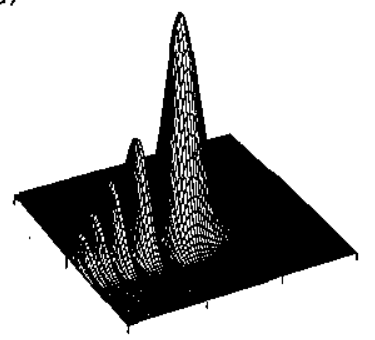

(b)

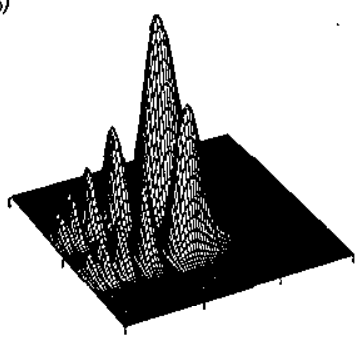

(c)

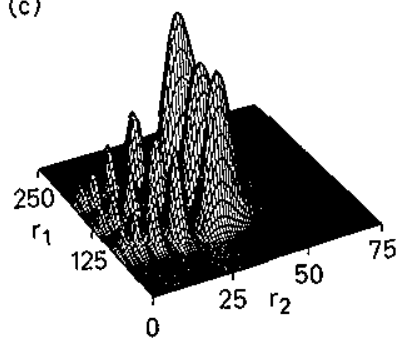

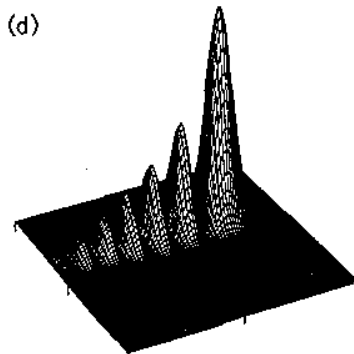

(d)

(e)
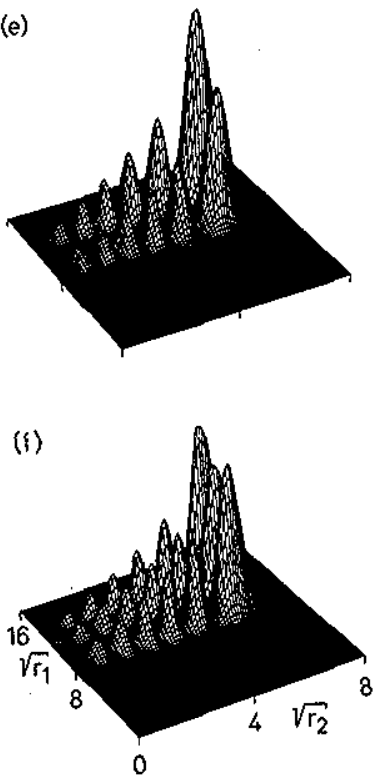

FIG. 7. Conditional probability densities of $(n, 0, l)$ frozen-planet states with $n=6$. The angle $\Theta$ between $r_{1}$ and $r_{2}$ is fixed to $\Theta=0$. The axes have a linear (left part) and a quadratic scale (right part), respectively. The states shown belong to $l=0(\mathrm{a}, \mathrm{d}), l=1(\mathrm{~b}, \mathrm{e})$, and $l=2(\mathrm{c}, \mathrm{f})$. Only the parts $r_{1}>r_{2}$ are shown. The full wave function is symmetric in $r_{1}$ and $r_{2}$. 
TABLE III. Coefficients $c_{j}$ of the cycle expansion, see Eq. (19) and text.

\begin{tabular}{lcccccc}
\hline & $\left|c_{0}\right|$ & $\left|c_{1}\right|$ & $\left|c_{2}\right|$ & $\left|c_{3}\right|$ & $\left|c_{4}\right|$ & $\left|c_{5}\right|$ \\
\hline$I_{\left(1+\alpha^{j} t_{\mathrm{PPO}}\right)}$ & 1 & 0.740 & 0.394 & 0.866 & 1.055 & 1.515 \\
$\mathrm{I}_{\left(1-\alpha^{j} t_{\mathrm{PPO}}\right)}$ & 1 & 0.740 & 0.394 & 0.283 & 0.204 & 1.937 \\
\hline \hline
\end{tabular}

tional probability distribution of the wave function for the $(6,0,0)$-state for the collinear arrangement $r_{12}=r_{1}-r_{2}$. The off-collinear part of the probability density, not shown here, decreases exponentially indicating a zero-point motion in the bending degree of freedom. This zero-point motion is expressed by the assignment $k=0$. The coordinate $r_{1}\left(r_{2}\right)$ denotes the radial distance of the outer (inner) electron. The outer electron probability is strongly localized in the region $r_{1} \approx 125$, reflecting the classical localization of the 'frozen' electron. Note also the large differences in the radial extents $r_{i}$. The nodal excitations are all directed along the frozen planet $\mathrm{PO}$, which is a nearly straight line along the frozen-planet radius indicated by an arrow in the figure. Recalling the typical quadratic spacing of nodal lines in Coulombic systems, we achieve nearly constant nodal distances by using quadratically scaled axes as done in part (d). The number of nodes along the orbit is 6 in agreement with the semiclassical predictions. The wave function only has a zero-point distribution perpendicular to the orbit (in the symmetry plane of collinear motion), which agrees with the semiclassical local coordinate classification $(n, k, l)=(6,0,0)$.

Wave functions with nodal excitations transverse to the orbit preserving the collinear character of the (quantum) motion are shown in parts (b), (e) and (c), (f) of Fig. 7. They correspond to the $l=1$ and $l=2$ nodal excitations of the $n=6$ manifold of states. Their energies differ only slightly due to the small winding number $\gamma_{R}$. A striking property of the wave functions shown is their nearly rectangular nodal structure and a near-separability in individual-particle coordinates $r_{1}, r_{2}$, i.e., the total wave function is approximately a product of wave functions for $r_{1}$ and $r_{2}$. It is widely accepted that two particles (or equivalently two degrees of freedom) are uncorrelated if the wave functions are products of single particle coordinates. Nevertheless, from our semiclassical analysis it is obvious that the radial motion of the electrons is highly correlated: it is the electron-electron interaction which is responsible for the dynamical localization of the outer electron. Thus Fig. 7 demonstrates that even if the motion is highly correlated the wave function may (approximately) separate in (independent) single-particle coordinates. It is only the other way round which generally holds: if the electrons are independent, then the wave functions separate in singleparticle coordinates.

\section{Quantizing chaotic dynamics: cycle expansion for near-collinear configurations}

The classical dynamics for collinear configurations with both electrons on different sides of the atoms turns out to be fully chaotic and the (approximate) torus quan- tization described in the previous section cannot be applied. We now have to sum over the contributions (9) of all periodic orbits. This leads to the so-called Gutzwiller trace formula, which for the present system reads ${ }^{7}$ (the product representation ${ }^{2,37}$ is used)

$$
\prod_{n}\left(E-E_{n}\right) \sim \prod_{\mathrm{PPO}} \prod_{k=0}^{\infty} \prod_{m=0}^{\infty}\left(1-t_{\mathrm{PPO}}^{(k, m)}\right) .
$$

The weight $t_{\mathrm{PPO}}^{(k, m)}$ of each PPO is given by

$$
\begin{aligned}
t_{\mathrm{PPO}}^{(k, m)}= & ( \pm 1)^{k} a^{j} \exp [2 \pi i z S-i \alpha \pi / 2 \\
& \left.-\left(k+\frac{1}{2}\right) u-4 \pi i\left(m+\frac{1}{2}\right) \gamma\right],
\end{aligned}
$$

where all classical quantities are given in Table I. The plus sign applies to hyperbolic PPO and the minus sign to hyperbolic PPO with reflection. The bookkeeping indices $a=1, j$ are only introduced for convenience and will be discussed below.

The formal expression (17) relates the product over quantum eigenvalues with a product over periodic orbits. Unfortunately, the zeros of the right hand side cannot be naively identified with the zeros of the left hand side, because the eigenvalues $E_{n}$ are located beyond the abscissa of absolute convergence of the rhs. ${ }^{38}$ The problem of finding semiclassical approximations for the energies $E_{n}$ from the diverging product over periodic orbits is a topic of several contributions to this issue of CHAOS and will not be discussed in detail here.

We use the cycle expansion ${ }^{23,39}$ to evaluate the semiclassical expression over PO. The idea of the cycle expansion is to expand the infinite product (17) into a power series $\Sigma_{j} c a^{j}$ of the bookkeeping index $a$. For $k=m$ $=0$ this reads ( $j$ equals the symbol length of the PO)

$$
\begin{aligned}
& \prod_{\mathrm{PPO}}\left(1-t_{\mathrm{PPO}}\right) \\
&=1-t_{+}-t_{-}-\left(t_{+-}-t_{+} t_{-}\right) \\
&-\left(t_{+}+-t_{+} t_{+-}\right)-\left(t_{+--}-t_{-} t_{+}\right) \\
&-\ldots
\end{aligned}
$$

Except for the fundamental orbits ' + ' and '- ' each orbit contribution is accompanied by a compensating term pieced together from shorter orbits. Thus terminating the expansion at a given symbol length effectively means a re-summation of all orbits, with the approximation that the longer orbits are shadowed to increasing accuracy by the shorter ones. The absolute convergence of the regrouped Dirichlet series (19) does of course not change. However, if each term $t_{a b}$ together with its shadowing term $t_{a} t_{b}$ is viewed as a single entry $d_{a b}$ then the series (19) 
TABLE IV. Total binding energies $E$ and effective quantum number $N_{\text {eff }}=1 / \sqrt{E}$ for ${ }^{1} S^{e}$ states obtained by WKB quantization of the fundamental orbit '- ', by the cycle expansion, and by full quantum solutions (taken from Ref. 7).

\begin{tabular}{|c|c|c|c|c|c|c|}
\hline \multirow[b]{2}{*}{$\left(n_{\lambda}, n_{\mu}\right)_{\nu}$} & \multirow[b]{2}{*}{$\left(N l, N^{\prime} l^{\prime}\right)$} & \multicolumn{3}{|c|}{$N_{\text {eff }}$} & \multicolumn{2}{|c|}{ Energies } \\
\hline & & WKB & Cycle & $\mathrm{QM}$ & Cycle & $\mathrm{QM}$ \\
\hline$(0,0)_{0}$ & $1 s 1 s$ & 0.568 & 0.584 & 0.587 & 2.932 & 2.904 \\
\hline$(0,2)_{0}$ & $2 s 2 s$ & 1.115 & 1.134 & 1.134 & 0.778 & 0.778 \\
\hline$(0,2)_{1}$ & $2 s 3 s$ & & 1.308 & 1.302 & 0.585 & 0.590 \\
\hline$(0,4)_{0}$ & $3 s 3 s$ & 1.662 & 1.684 & 1.682 & 0.353 & 0.354 \\
\hline$(0,4)_{1}$ & $3 s 4 s$ & & 1.883 & 1.886 & 0.282 & 0.281 \\
\hline$(0,6)_{0}$ & $4 s 4 s$ & 2.208 & 2.243 & 2.231 & 0.199 & 0.201 \\
\hline$(0,6)_{1}$ & $4 s 5 s$ & & 2.456 & 2.456 & 0.166 & 0.166 \\
\hline$(0,6)_{2}$ & $4 s 6 s$ & & 2.574 & 2.575 & 0.151 & 0.151 \\
\hline$(0,8)_{0}$ & $5 s 5 s$ & 2.755 & 2.783 & 2.780 & 0.129 & 0.129 \\
\hline$(0,8)_{1}$ & $5 s 6 s$ & & 3.025 & 3.020 & 0.109 & 0.110 \\
\hline$(0,8)_{2}$ & $5 s 7 s$ & & 3.154 & 3.159 & 0.101 & 0.100 \\
\hline$(0,10)_{0}$ & $6 s 6 s$ & 3.302 & 3.343 & 3.329 & 0.0895 & 0.0902 \\
\hline$(0,10)_{1}$ & $6 s 7 s$ & & 3.586 & 3.580 & 0.0778 & 0.0780 \\
\hline$(0,10)_{2}$ & $6 s 8 s$ & & 3.733 & 3.733 & 0.0717 & 0.0718 \\
\hline$(0,12)_{0}$ & $7 s 7 s$ & 3.849 & 3.903 & 3.883 & 0.0657 & 0.0663 \\
\hline$(0,12)_{1}$ & $7 s 8 s$ & & 4.140 & 4.138 & 0.0583 & 0.0584 \\
\hline$(0,12)_{2}$ & $7 s 9 s$ & & 4.305 & 4.301 & 0.0540 & 0.0541 \\
\hline$(0,14)_{0}$ & $8 s 8 s$ & 4.395 & 4.429 & 4.411 & 0.0510 & 0.0514 \\
\hline$(0,14)_{1}$ & $8 s 9 s$ & & 4.689 & 4.686 & 0.0455 & 0.0455 \\
\hline$(0,14)_{2}$ & $8 s 10 s$ & & 4.865 & 4.865 & 0.0423 & 0.0423 \\
\hline
\end{tabular}

converges absolutely. This is illustrated in Table III which shows the coefficients $c_{j}$ of the cycle expanded product ( $k=m=0$ ) with the semiclassical weights replaced by their absolute values, i.e. $t_{\mathrm{PPO}}=\exp (-\lambda / 2)$. Obviously, the coefficients of the first row (determining the abscissa of absolute convergence of the unexpanded product) diverge exponentially with a ratio $c_{j+1} / c_{j} \approx 1.3$, whereas the coefficients of the second row converge exponentially with a ratio $c_{j+1} / c_{j} \approx 0.65$. A more careful analysis shows that the unexpanded product converges absolutely for $\Im \sqrt{-E}$ $>0.027$, i.e. only sufficiently far in the upper half of the complex energy plane, whereas the resonance poles are located close to the real energy axis. The cycle expansion, however, converges in the energy region, where the resonances are located (typically $\Im \sqrt{-E}>-0.01$ ).

The products over $k$ and $m$ in Eq. (17) originate from the expansion of the Gutzwiller amplitudes (9) into geometric series. ${ }^{37}$ They have to be treated differently, because the stability characteristic is different for the two directions perpendicular to the orbit. Similar to the treatment for the (doubly) stable frozen planet orbit we identify $m$ as a semiclassical quantum number for the stable bending degree of freedom. For the expansion of the remaining product we set the bookkeeping index $j$ to $(2 k+1)$ times the symbol length of the PO. The present calculations are carried out including all orbit contributions up to $j=6$. In Table IV we show our results for some doubly excited ${ }^{1} S^{e}$ states with $m=0$. For labeling the states we use the molecularorbital (MO) classification $\left(n_{\lambda}, n_{\mu}\right)_{v}$ derived from an adiabatic treatment of the inter-electron vector $\mathbf{R}^{40}$ The MO quantum numbers accurately describe the nodal surfaces of the quantal wave functions for fixed inter-electron distances and moderate electron excitations $\left(N, N^{\prime}<6\right) .{ }^{41,42}$ For convenience, we also give the "inde- pendent particle" labeling $\left(N l, N^{\prime} l^{\prime}\right)$, i.e. the configuration which comes closest in an independent particle description. Here, $N, N^{\prime} \geqslant N$ roughly correspond to the principal quantum numbers of the electrons.

It is more natural to compare the effective quantum numbers $N_{\text {eff }}=E^{-1 / 2}$ than the binding energies $E$ themselves, but both values are given in the table. We find that the cycle expansion results are mostly good to within $1 \%$ or better. This is better than might be expected; in fact, accurate quantum results for the very high lying doubly excited states $(N>6)$ are presently not available in the literature. For published data the results are comparable or even superior to elaborate (adiabatic) hyperspherical calculations. ${ }^{43,44}$

Generally, the energies obtained in the cycle expansion (as well as in the quantum mechanical calculations) are complex valued and we have only tabulated the real part of these energies. The widths of the resonances (i.e. the imaginary part of their energies) are still smaller than the semiclassical error in the real part and there is no likely reason why the imaginary part should be more accurate.

One may wonder why the semiclassical analysis works fine even down to the ground state, which has a smooth and-more or less-structureless wave function. The reason is that the PO do not only contain the information about the underlying dynamics, but they also "know" the size of the phase space. It is this property which often leads to rather good results when a fundamental (e.g. the shortest) orbit is naively quantized.

Although the results for the semiclassical energies are very satisfactory, it is even more valuable that the (semi-) classical analysis provides an insight of what the electrons are actually 'doing' in the highly correlated states. In Table IV we also list the results of the simplest cycle approach 


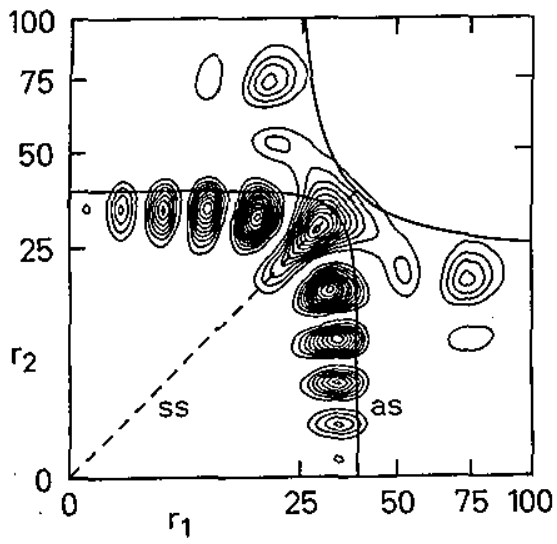

FIG. 8. Contour plot of the conditional probability distribution $\left|\Psi_{N N^{\prime}}\left(r_{1}, r_{2}, r_{12}=r_{1}+r_{2}\right)\right|^{2}$ for the intra-shell wave function $N=N^{\prime}$ $=6$ corresponding to the collinear arrangement $\Theta=\pi$ of the electrons. The axes have a quadratic scale to account for the wave propagation in Coulombic systems, where nodal distances increase quadratically. The fundamental orbit " -' (AS) as well as the symmetric stretch motion (SS) along the Wannier ridge are overlaid.

including only the fundamental PO ' - ', which is nothing but a WKB quantization of the orbit (but including the zero-point motion for the perpendicular degrees of freedom). Quantization of this fundamental asymmetric stretch PO gives rather accurate results for the doubly excited intra-shell resonances $N=N^{\prime}$. For the other states $N \neq N^{\prime}$ the inclusion of all the orbits of Table I is essential and the simplified WKB approach cannot yield them. These results indicate that the intra-shell resonances are associated with the asymmetric stretch like motion of the fundamental PO ' - ' rather than the symmetric stretch motion along the Wannier ridge. This conclusion is in striking contrast to the common viewpoint expressed in the literature (see, e.g., Refs. 25-30), but in line with recent suggestions. ${ }^{5,8,42}$

An inspection of the quantum mechanical intra-shell wave functions confirms the semiclassical conclusion on the fundamental electron motion. In Fig. 8 we show, e.g., the probability distribution of the $N=N^{\prime}=6$ state which is clearly localized along the fundamental orbit " - ' (AS) and not along the Wannier ridge $r_{1} \equiv r_{2}$ (SS). The classical probability along the trajectory is largest (as well as the quantal wave function) where it passes the Wannier saddle point, but the motion is directed perpendicular to the ridge.

The WKB treatment of the fundamental orbit also provides the dynamical origin of the double Rydberg formula ${ }^{26}$

$$
E_{N}=-(Z-\sigma)^{2} /(N-\mu)^{2}
$$

for the intra-shell resonances. We find $Z-\sigma=S_{-}$and $\mu=1-\gamma_{-}-\alpha_{-} / 4$ (i.e. $\sigma=0.1710$ and $\mu=$ - 0.0393) which fits well with the semi-empirically derived values of $\sigma=0.1795$ and $\mu=-0.0597 .^{45}$

The classical analysis also applies to the ${ }^{3} S^{e}$ states, i.e. those which are anti-symmetric with respect to the exchange of particle (configuration space) coordinates. For the semiclassical Green function we now have to take the Dirichlet boundary condition along the symmetry line $r_{1} \equiv r_{2}$ instead of the von Neumann boundary condition. Thus each time a trajectory crosses the symmetry line we have an additional phase loss of $\pi$. Again the coding takes care automatically of the additional total phase loss: each symbol ' - ' of the electron pair motion is associated with a crossing of the symmetry line.

Table $\mathrm{V}$ gives the results for the low-lying 'intra-shell' ${ }^{3} S^{e}$ states. The overall agreement with the quantum results is again remarkable. From the (semi-) classical analysis one expects the states to be of similar nature as those of ${ }^{1} S^{e}$ symmetry. This is verified in Fig. 9 for the state $\left(n_{\lambda}, n_{\mu}\right)_{v}=(0,11,0)_{0}$. Obviously, the wave function images the same type of electron pair motion as Fig. 8 for the ${ }^{1} S^{e}$ symmetry. The only difference is that the wave function has an odd number of nodes along the $\mathrm{PO}$ and hence a node at $r_{1}=r_{2}$. The wave functions shown are completely symmetric with respect to 'individual' electron excitations. This demonstrates that the independent particle label $\left(N l, N^{\prime} l^{\prime}\right)=6 s 7 s$ [as well as the popular $N(K, T)^{A} N^{\prime}$ labeling scheme $\left.{ }^{27}\right]$ is not useful classifying the internal structure of the state shown in Fig. 9. The MO quantum numbers, however, do give a proper classification of the state. The number of nodes along the PO is given by $n_{\mu}$, whereas nodal excitations perpendicular to the orbit are labeled by $v$. The quantum number $n_{\lambda}$ describes the bending degree of freedom and is identical to the semiclassical quantum number $m$ (both of which are zero for the states discussed so

TABLE V. Same as Table IV, but for ${ }^{3} S^{e}$ states.

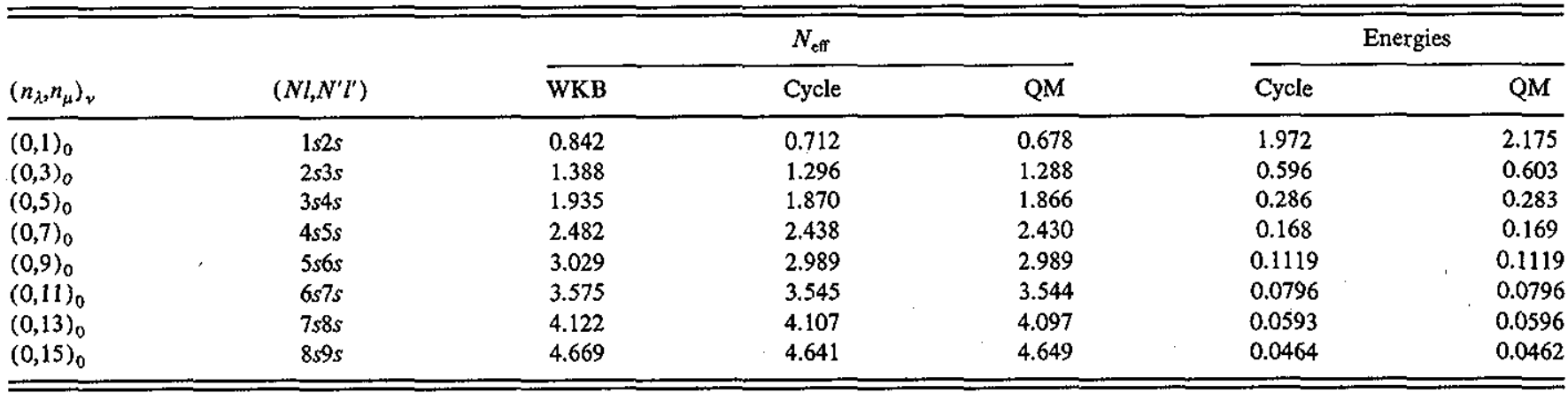




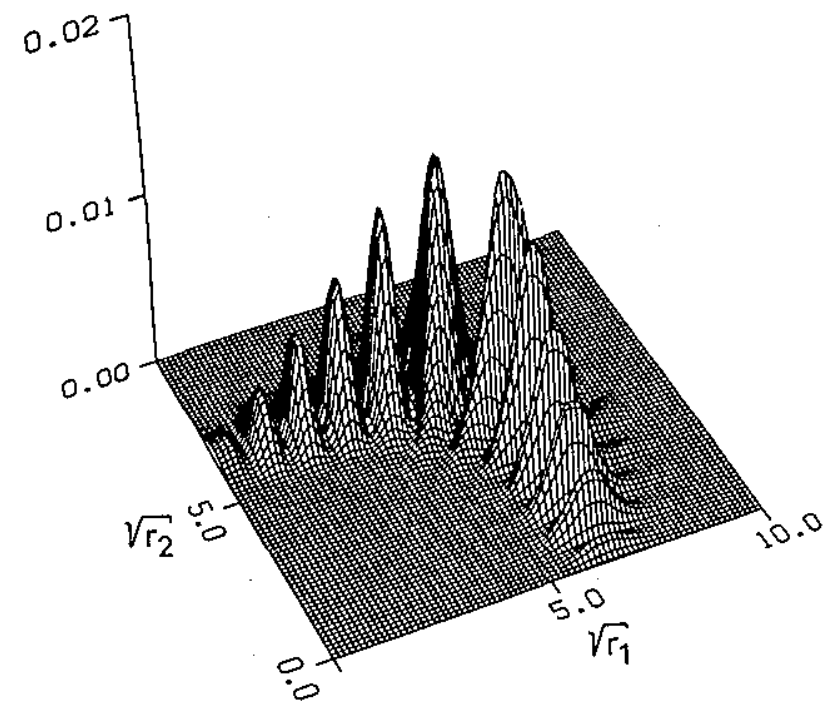

FIG. 9. Conditional probability distribution $\left|\Psi_{N N^{\prime}}\left(r_{1}, r_{2}, r_{12}=r_{1}+r_{2}\right)\right|^{2}$ for the wave function $\left(n_{\lambda}, n_{\mu}\right)_{\nu}=(0,11)_{0}$.

far). In addition, for fixed inter-electron distance $r_{12}$ the internal wave function approximately separates in MO coordinates. ${ }^{42}$

The choice $m=0$ in our calculations means that the associated semiclassical wave functions are localized in the symmetry plane of collinear motion with only a zero-point motion perpendicular to it. This approach is justified by the stability of the classical motion perpendicular to the plane. In fact, the quantum wave functions show the same behavior. Putting $m \neq 0$ gives states with $n_{\lambda}=m$ in the MO description, but the linearization of the motion perpendicular to the plane becomes a rather crude approximation if $N \gg m$ does not hold. The results for intra-shell states of ${ }^{1} S^{e}$ symmetry are summarized in Table VI . The semiclassical error is about $10 \%$ for the lowest lying state, for which there is no nodal excitation along the PO but one nodal excitation perpendicular to the symmetry plane. For higher lying states the semiclassical error again drops below $0.5 \%$.

\section{ADIABATIC VERSUS CHAOTIC MOTION}

Typically, classical chaotic motion is connected with the loss of (approximate) symmetries and associated quantum numbers. ${ }^{46,47}$ One may then wonder, why all the states reported in this contribution can still be labeled with ap- proximate quantum numbers, even though we exploited the intrinsic chaotic classical dynamics to calculate semiclassical eigenvalues.

Different sets of approximate quantum numbers describing three-body Coulomb systems were derived, partly starting from the independent particle description and applying degenerate perturbation theory for the electronelectron interaction. ${ }^{48}$ More sophisticated perturbative calculations include group-theoretical methods. ${ }^{49}$ Adiabatic expansions using the hyperspherical radius $\mathscr{R}{ }^{25,27}$ or the inter-electron radius $R=r_{12}$ (Refs. 40,50 ) as adiabatic coordinates have been proposed to describe the internal structure of doubly excited states. However, since there is no obvious geometrical or kinematical reason, there are only few justifications why these coordinates can be treated adiabatically. Nevertheless, energies derived from both adiabatic approaches, hyperspherical and MO, yield quite accurate results for the states of maximal polarization along the inter-electron axis, i.e. those states for which - $\langle\cos \Theta\rangle$ is close to unity. ${ }^{28,30,40,43,44}$ However, energies are not a sensitive test of the validity of the underlying assumptions. For example, highly accurate energy values for the intra-shell resonances can also be obtained within a diabatic approach, ${ }^{51}$ even, though the corresponding wave functions are totally inappropriate to describe the nodal structure of the states.

The possibility of an adiabatic description of the (regular) frozen planet configurations is immediately obvious from the consideration of the classical motion of the electrons. For the frozen planet PO the action of the accumulated action of the outer electron is about $2 \times 10^{4}$ smaller than the action of the inner electron. Since the frozen electron is strongly localized in configuration space, the outer electron radius $r_{1}$ suggests itself as an adiabatic coordinate. For fixed $r_{1}$ the remaining inner electron Hamiltonian is separable in (molecular) prolate spheroidal coordinates, which allows the labeling of the resulting adiabatic potential curves with a complete set of quantum numbers. For two-electron atoms the goodness of the adiabatic approximation for the frozen planet configurations has been analyzed in Ref. 52. If the outer electron is replaced by a heavy particle of the same charge (e.g. an anti-proton) then the adiabatic approach should be even more efficient. The antiprotonic analog of the frozen planet states were proposed recently as possible anti-matter traps. ${ }^{53,54}$ The overlap of the anti-protonic wave function with the nucleus is extremely small, which prevents the three-body complex from decay via the strong interaction.

TABLE VI. Same as Table IV, but for ${ }^{1} S^{e}$ states with $n_{\lambda}=1$.

\begin{tabular}{|c|c|c|c|c|}
\hline \multirow[b]{2}{*}{$\left(n_{\lambda}, n_{\mu}\right)_{\nu}$} & \multirow[b]{2}{*}{$\left(N l, N^{\prime} l^{\prime}\right)$} & \multicolumn{3}{|c|}{ Energies } \\
\hline & & WKB & Cycle & $\mathrm{QM}$ \\
\hline$(1,0)_{0}$ & $2 p 2 p$ & 0.745 & 0.701 & 0.622 \\
\hline$(1,2)_{0}$ & $3 p 3 p$ & 0.344 & 0.337 & 0.317 \\
\hline$(1,4)_{0}$ & $4 p 4 p$ & 0.197 & 0.191 & 0.188 \\
\hline$(1,6)_{0}$ & $5 p 5 p$ & 0.1277 & 0.1260 & 0.1233 \\
\hline$(1,8)_{0}$ & $6 p 6 p$ & 0.0894 & 0.0873 & 0.0869 \\
\hline
\end{tabular}



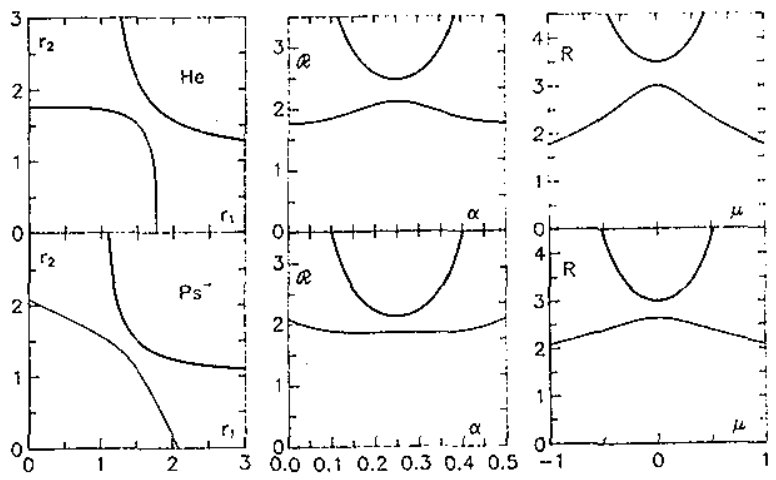

FIG. 10. The asymmetric stretch-type $\mathrm{PO}^{\prime}-{ }^{\prime}$ ' in individual particle coordinates $r_{1}, r_{2}$, hyperspherical coordinates $\mathscr{R}=\left(r_{1}+r_{2}\right)^{1 / 2}, \alpha$ $=\operatorname{atan}\left(r_{1} / r_{2}\right) / \pi$, and MO-coordinates $R=r_{12}, \mu=\left(r_{1}-r_{2}\right) / R$. The boundaries of the classically allowed regions are drawn as thick lines. The upper half of the figure applies to helium $(\mathrm{He})$, the lower part to the positronium negative ion ( $P_{\mathrm{s}}{ }^{-}$). For a better comparison $Z$-scaled coordinates $r_{i} / Z$ are used.

An adiabatic description of intra-shell states or nearcollinear configurations with both electrons on opposite sides is not immediately obvious. Recently it has been shown however, ${ }^{41,42}$ that the nodal structure of (moderately) doubly excited intra-shell states is accurately described by the MO-quantum numbers derived from an adiabatic treatment of the inter-electron distance. Again, for fixed $\mathbf{R}=\mathbf{r}_{1}-\mathbf{r}_{2}$ the electronic center of mass (ECM) coordinate $\mathbf{r}=\left(\mathbf{r}_{1}+\mathbf{r}_{2}\right) / 2$ separates in molecular coordinates ${ }^{50}$ leading to the full set of MO-quantum numbers used in Tables IV-VI. The adiabatic treatment does not imply that the actual wave functions separate (even approximately) in $R$ and $\mathbf{r}$; the nodal structure of the full wave function may show strong mixing of the ECMcoordinates with $R$ (which actually is the case ${ }^{42}$ for the intra-shell resonances discussed here). To illustrate this consider the wave function

$$
\Psi(R, x, y)=\phi_{R}(R) \phi_{x}(R x) \phi_{y}(R y) .
$$

For each valuc of $R$ the wave function separates exactly in $x, y$, leading to a rectangular nodal pattern in $x$ and $y$. The full wave function, however, mixes $R$ with $x$ and $y$. The wave function (21) may be perfectly adiabatic, nevertheless it is non-separable in full space.

The classical analysis of collinear electron configurations and the subsequent semiclassical treatment with the cycle expansion uncovers the asymmetric stretch-type PO '- ' as the fundamental electron motion for the intra-shell states of helium. Figure 10 shows the PO in the different adiabatic coordinate sets discussed above. For all the three coordinate sets the symmetry plane of collinear configuration results in a constant value of one coordinate, $\Theta \equiv \pi$ for the individual and hyperspherical coordinates, and $\lambda$ $=\left(r_{1}+r_{2}\right) / R \equiv 1$ for the MO-coordinates. Even though the individual radial distances $r_{1}, r_{2}$ vary largely, both the hyperradius $\mathscr{R}$ and the inter-electron distance $R$ change only slightly along the trajectory. These results may ex- plain to some extent why the intra-shell resonances can be treated adiabatically in $\mathscr{R}$ or $R$ to good approximation.

The adiabatic behavior becomes even more pronounced for the molecular-like positronium negative ion $\left(\mathrm{Ps}^{-}=e^{-} e^{+} e^{-}\right)$, as is shown in the lower half of Fig. 10. The fundamental $\mathrm{PO}$ ' - ' is stable for $\mathrm{Ps}^{-}$, even though the stability island surrounding the orbit is rather small. From the shape of the fundamental PO in MOcoordinates, it is not too surprising, that the results of adiabatic molecular quantum calculations are rather accurate in this case. ${ }^{55}$

The adiabatic behavior is somewhat hidden in the classical/semiclassical treatment described in the previous sections. As we have seen, the WKB-quantization of the fundamental $\mathrm{PO}$ ' - ' accounts for the intra-shell resonances $N=N^{\prime}$, which is the first member of a Rydberg series $N^{\prime} \geqslant N$ of resonances converging to the singleparticle escape threshold leaving the $\mathrm{He}^{+}$-ion in a Starktype polarized hydrogenic state with principal quantum number $n=N$. For most of the states considered here $(N \leqslant 8)$, the energy differences between the exterior scaling $N$ and the interior Rydberg scaling $N^{\prime}$ are rather large, which, translated via the correspondence principal, leads to largely different time scales in the system. But as can be seen from Table I the distribution of periods (actions) of the PO is rather smooth. How do the PO then reflect the different time scales? Amusingly, it is the nearly perfect hyperbolicity of the system which is responsible for the regularity (and thus adiabaticity) of the spectra. The inclusion of more and more orbits in the cycle expansion does not destroy the exterior scaling, which is already described by the fundamental PO '- '. The longer orbits are nearly perfectly shadowed by the compensating terms of shorter orbits, i.e.

$$
t_{a b}-t_{a} t_{b}=t_{a b}\left\{1-\exp \left[2 \pi i z\left(S_{a}+S_{b}-S_{a b}\right)\right.\right.
$$

$$
\left.\left.-\left(u_{a}+u_{b}-u_{a b}\right) / 2\right]\right\} \approx 0 .
$$

The relevant quantities are then the small differences in the periods of the orbits and their shadowing parts. Inclusion of these contributions yield the other members of the Rydberg series tabulated in Table IV.

The energetic separation of the different Rydberg series for $N<6$ combined with the quasi-separability of the bending degree of freedom is the reason why approximate quantum numbers exist. Due to the energetic separation, wave functions cannot mix with those originating from other series and they become rather simply structured similar to what one would expect for 'regular' wave functions. Near $N \geqslant 6$ the different Rydberg series begin to overlap energetically, and it is this region where we expect the breakdown of approximate quantum numbers and the appearance of irregular spectra and wave functions, reflecting the intrinsic chaotic dynamics of the electron motion (see also Ref. 9). 


\section{SUMMARY AND CONCLUSIONS}

At present, semiclassical theories undergo a rapid and exciting evolution. The present issue of CHAOS documents part of this development. In this contribution we applied many of the new ideas to the problem of twoelectron atoms.

The classical dynamics of the collinear helium atom with both electrons on different sides is fully chaotic. An application of the Gutzwiller formula for the full threedimensional problem combined with the cycle expansion yields a number of resonances with high accuracy. The interpretative ability of the methods illuminates the structure of the quantal motion. The analysis shows that the near-collinear intra-shell resonances are associated with the (fundamental) asymmetric stretch like motion of the electron pair. Semiclassically, this observation is nearly trivial. The result is nevertheless remarkable, in that it has been widely believed for decades that these resonances are associated with the in-phase symmetric stretch motion of the electron pair along the Wannier ridge.

The classical dynamics of the collinear helium atom with both electrons on the same side of the nucleus is fully stable. Approximate torus quantization yields very accurate results for the positions of the associated quantum mechanical resonances. The semiclassical formalism also accounts semi-quantitatively for the decay widths and degeneracies of doublet states. The structure of the wave functions corresponds to what one would expect from considering the classical motion.

We are certainly at the beginning of refining the semiclassical methods for multi-dimensional methods like the PO-quantization approach. Important further developments will probably include the description of dynamical tunneling processes and a refined semiclassical consideration of discrete symmetries, which may allow the calculation of exponentially small decay widths or multipletdegeneracies. An important (even though presumably formidable) step would be the inclusion of higher order terms of $\hbar$ in the semiclassical PO-theory. Other methods than the cycle expansion which drastically reduce the (exponentially growing) classical input of the semiclassic POquantization are also highly desirable. Even without such refinements, it is likely that the combined classical/ semiclassical analysis of few-body systems such as the helium atom will uncover some more surprises in the near future.

\section{ACKNOWLEDGMENTS}

We gratefully acknowledge the collaboration with G. S. Ezra, from which parts of the presented results originate. We thank J. S. Briggs and J.-M. Rost for many discussions on three-body Coulomb problems, and P. Cvitanović and B. Eckhardt for motivating us to "cycle tours." The research was supported by the Deutsche Forschungsgemeinschaft (Wi877/2 and Wi877/5), and partly by the SFB 276 located in Freiburg and by NORDITA in Copenhagen.
${ }^{1}$ J. H. van Vleck, Philos. Mag. 44, 842 (1922).

${ }^{2}$ M. C. Gutzwiller, Chaos in. Classical and Quantum Mechanics (Springer, New York, 1990).

${ }^{3}$ M. Born, Vorlesungen über Atommechanik (Springer, Berlin, 1925). English translation: The Mechanics of the Atom (Ungar, New York, 1927).

${ }^{4}$ J. G. Leopold and I. C. Percival, J. Phys. B 13, 1037 (1980).

${ }^{5} \mathrm{~K}$. Richter and D. Wintgen, J. Phys. B 23, L197 (1990).

${ }^{6}$ K. Richter and D. Wintgen, Phys. Rev. Lett. 65, 1965 (1990); J. Phys. B 24, (1991), L565.

${ }^{7}$ G. S. Ezra, K. Richter, G. Tanner, and D. Wintgen, J. Phys. B 24, L413 (1991).

${ }^{8}$ J.-H. Kim and G. S. Ezra, in Proceeding of the Adriatico Conference on Quantum Chaos, edited by H. A. Cerdeira et al. (World Scientific, Singapore, 1991).

${ }^{9}$ R. Blümel and W. P. Reinhardt, in Directions in Chaos, edited by B. L. Hao et al. (World Scientific, Hong Kong, 1991), Vol. 4.

${ }^{10}$ B. Eckhardt, preprint Universität Marburg; Habilitationsschrift, Universität Marburg (1991).

"J. Müller, J. Burgdörfer, and D. W. Noid, preprint University of Tennessee.

${ }^{12}$ C. Marchal, The Three-Body Problem (Elsevier, Amsterdam, 1990).

${ }^{13}$ C. L. Siegel, Ann. Math. 42, 127 (1941).

${ }^{14}$ H. M. James and A. S. Coolidge, Phys. Rev. 51, 857 (1937).

${ }^{15}$ G. Wannier, Phys. Rev. 90, 817 (1953).

${ }^{16}$ M. Domke, C. Xue, A. Puschmann, T. Mandel, E. Hudson, D. A. Shirley, G. Kaindl, C. H. Greene, H. R. Sadeghpour, and H. Petersen, Phys. Rev. Lett. 66, 1306 (1991).

${ }^{17}$ B. Eckhardt and D. Wintgen, J. Phys. B 23, 355 (1990).

${ }^{18}$ R. Schinke and V. Engel, J. Chem. Phys. 93, 3252 (1990).

${ }^{19}$ P. Dahlquist and G. Russberg, J. Phys. A 24, 4763 (1991).

${ }^{20}$ D. Weiss, M. L. Roudes, A. Menschig, P. Grambow, K, von Klitzing, and G. Weimann, Phys. Rev. Lett. 66, 2790 (1991).

${ }^{21} \mathrm{M}$. Sieber and F. Steiner, Phys. Lett. A148, 415 (1990); M. Sieber, Dissertation, Universität Hamburg (1991).

${ }^{22} \mathrm{G}$. Tanner and D. Wintgen, Chaos 2, 53-59 (1992).

${ }^{23}$ P. Cvitanović and B. Eckhardt, Phys. Rev. Lett. 63, 823 (1989).

${ }^{24}$ B. Eckhardt and D. Wintgen, J. Phys. A 24, 4335 (1991).

${ }^{25}$ U. Fano, Phys. Rep. 46, 97 (1983).

${ }^{26}$ A. R. P. Rau, J. Phys. B 16, L699 (1983).

${ }^{27}$ S. Watanabe and C. D. Lin, Phys. Rev. A 34, 823 (1986).

${ }^{28}$ H. R. Sadeghpour and C. H. Greene, Phys. Rev. Lett. 65, 313 (1990).

${ }^{29}$ P. G. Harris, H. C. Bryant, A. H. Mohagheghi, R. A. Reeder, C. Y. Tang, J. B. Donahue, and C. R. Quick, Phys. Rev. A 42, 6443 (1990).

${ }^{30}$ H. R. Sadeghpour, Phys. Rev. A 43, 5821 (1991).

${ }^{31}$ M. C. Gutzwiller, J. Math. Phys. 8, 1979 (1967); 10, 1004 (1969); 11, 1791 (1970); 12, 343 (1971).

${ }^{32}$ M. V. Berry and K. T. Mount, Rep. Prog. Phys. 35, 315 (1972).

${ }^{33}$ A. Voros and B. Grammaticos, Ann. Phys. (NY) 123, 359 (1979).

${ }^{34}$ W. H. Miller, J. Chem. Phys. 63, 996 (1975).

${ }^{35}$ E. B. Bogomolny, Physica D (Amsterdam) 31, 169 (1988).

${ }^{36}$ M. J. Davies and E. J. Heller, J. Chem. Phys. 75, 246 (1981).

${ }^{37}$ A. Voros, J. Phys. A 21, 685 (1988).

${ }^{38}$ B. Eckhardt and E. Aurell, Europhys. Lett. 9, 509 (1989).

${ }^{39}$ R. Artuso, E. Aurell, and P. Cvitanović, Nonlinearity 3, 325 and 361 (1990).

${ }^{40}$ J. M. Rost and J. S. Briggs, J. Phys. B 24, 4293 (1991).

${ }^{41}$ J. M. Rost, J. S. Briggs, and J. M. Feagin, Phys. Rev. Lett. 66, 1642 (1991).

${ }^{42}$ J. M. Rost, R. Gersbacher, K. Richter, J. S. Briggs, and D. Wintgen, J. Phys. B 24, 2455 (1991).

${ }^{43}$ N. Koyama, H. Fukuda, T. Motoyama, and M. Matsuzawa, J. Phys. B 19, L331 (1986).

${ }^{44}$ H. Fukuda, N. Koyoma, and M. Matsuzawa, J. Phys. B 20, 2959 (1987).

${ }^{45}$ Q. Molina, Phys. Rev. A 39, 3298 (1989).

${ }^{46}$ B. Eckhardt, Phys. Rep. 163, 205 (1988).

${ }^{47}$ H. Friedrich and D. Wintgen, Phys. Rep. 183, 37 (1989).

${ }^{48}$ U. Fano and A. R. P. Rau, Atomic Collisions and Spectra (Academic, London, 1986).

${ }^{49}$ D. E. Herrick, Adv. Chem. Phys. 52, 1 (1983).

${ }^{50}$ J. M. Feagin and J. S. Briggs, Phys. Rev. Lett. 57, 984 (1986); Phys. Rev. A 37, 4599 (1988).

${ }^{51}$ J. M. Rost and J. S. Briggs, J. Phys. B 22, 3587 (1989). 
${ }^{52} \mathrm{~K}$. Richter, J. S. Briggs, D. Wintgen, and E. A. Solov'ev, submitted to J. Phys. B.

${ }^{53}$ K. Richter, J. M. Rost, R. Thürwächter, J. S. Briggs, D. Wintgen, and E. A. Solov'ev, Phys. Rev. Lett. 66, 149 (1991).
${ }^{54}$ M. Iwasaki, S. N. Nakamura, K. Shigaki, Y. Shimizu, H. Tamura, T. Ishikawa, R. S. Hayano, E. Takado, E. Widmann, H. Outa, M. Aoki, P. Kitching, and T. Yamazaki, Phys. Rev. Lett. 67, 1246, (1991).

${ }^{55} \mathrm{~J}$. M. Rost and D. Wintgen (in preparation). 